\title{
Dietary effects on mRNA levels of stearoyl-CoA desaturase isoforms in the hamster
}

\author{
Catherine Major, Thomas Vallim and Andrew Salter \\ School of Biosciences, University of Nottingham, Loughborough, Leicestershire, UK
}

Stearoyl-CoA desaturase (SCD) introduces a double bond at the $\Delta 9$ position of various fatty acids. The enzyme's primary function appears to be the conversion of stearic acid to oleic acid (cis-9 18:1), the predominant MUFA in the body. Reduction of SCD activity has been shown to result in reduction of body fat in mice, whereas, increased SCD levels have been found to be increased in obese mouse models $^{(1)}$. The Golden Syrian hamster has been shown to exhibit a number of advantages compared with other small-animal models of lipid and lipoprotein metabolism. While four isoforms of SCD have been reported in mice (each of which show specific tissue expression and response to nutritional factors), until recently only one isoform had been described in the hamster. However, a recent report has suggested that this species expresses at least three isoforms analogous to SCD1, SCD2 and SCD3 of the mouse ${ }^{(2)}$. The present experiment has investigated the effects of dietary fat and cholesterol (chol) on the expression and abundance of the three SCD isoforms in hamster liver and white adipose tissue.

Male Golden Syrian hamsters (eight per group) were fed a low-fat chow-based diet (chow) alone or with $0.2 \%$ (w/w) chol or $15 \%$ (w/w) added fat (formulated to represent the fatty acid composition of a typical Western diet) or both. The diets were fed for 4 weeks, after which the animals were killed and the liver and adipose tissues were removed, snap-frozen in liquid $\mathrm{N}_{2}$ and stored at $-80^{\circ} \mathrm{C}$ until required for determination of mRNA concentration. The steady-state mRNA concentration for all three SCD isoforms and TATA box-

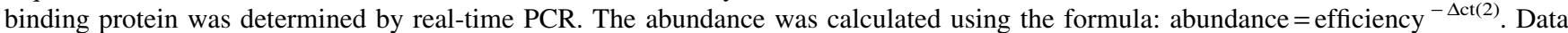
were analysed by two-way ANOVA, exploring significant effects of chol, fat or an interaction between the two (chol $\times$ fat).

SCD1 was the major isoform expressed in both liver and adipose tissue, with its expression in the liver being approximately 50-fold higher than SCD3 and 300-fold higher than SCD2. Diet had little impact on the expression of any of the isoforms in adipose tissue. However, specific effects of diet were seen on the relative abundance (arbitrary units) of each of the isomers in the liver (Table).

\begin{tabular}{|c|c|c|c|c|c|c|c|}
\hline \multirow{2}{*}{$\begin{array}{l}\text { Diet... } \\
\text { SCD1 }\end{array}$} & \multirow{2}{*}{$\begin{array}{l}\text { Chow } \\
244.2\end{array}$} & \multirow{2}{*}{$\begin{array}{l}\text { + Chol } \\
233.9\end{array}$} & \multirow{2}{*}{$\frac{\text { + Fat }}{35.2}$} & \multirow{2}{*}{$\begin{array}{c}+ \text { Chol + fat } \\
149.3\end{array}$} & \multirow{2}{*}{$\begin{array}{r}\text { SED } \\
28.0\end{array}$} & \multicolumn{2}{|c|}{$P$ for the effect of: } \\
\hline & & & & & & Chol & 0.014 \\
\hline & & & & & & Fat & $<0.001$ \\
\hline & & & & & & Chol $\times$ fat & 0.004 \\
\hline \multirow{3}{*}{$\mathrm{SCD} 2$} & 0.734 & 0.902 & 0.582 & 1.316 & 0.15 & Chol & $<0.001$ \\
\hline & & & & & & Fat & 0.214 \\
\hline & & & & & & Chol $\times$ fat & 0.011 \\
\hline \multirow[t]{3}{*}{$\mathrm{SCD} 3$} & 5.6 & 9.9 & 5.2 & 10.9 & 1.8 & Chol & $<0.001$ \\
\hline & & & & & & Fat & 0.823 \\
\hline & & & & & & Chol $\times$ fat & 0.591 \\
\hline
\end{tabular}

Dietary chol alone had no significant effect on SCD1 or SCD2 expression but significantly increased the expression of SCD3. By contrast, dietary fat reduced the expression of SCD1 but did not affect SCD3. Adding chol to fat-rich diets partly reversed the effects of fat alone on SCD1 and SCD2 expression. Feeding the combined diet significantly reduced the abundance of both SCD1 and SCD2. SCD3 abundance in the liver was shown to be increased by feeding chol compared with chow and fat - rich diets.

The results confirm that, as in the mouse, SCD1 is the predominant isoform in the liver and adipose tissue of the hamster. The hepatic expression of all three isoforms is influenced by dietary lipids, with the most pronounced effect being a down-regulation of SCD1 by a diet rich in saturated fat, which is partly reversed by dietary chol. A better understanding of the mechanisms whereby dietary lipids regulate SCD expression should provide insights into how its expression and activity can be manipulate to regulate tissue fatty acid composition and adipose tissue deposition.

This work was supported by the British Heart Foundation.

1. Ntambi JM \& Miyazaki M (2004) Prog Lipid Res 43, 91-104

2. Jian W, Lan Y, Wang H et al. (2008) Lipids 43, 197-205. 